\title{
REFERENCES
}

1. P. R. Halmos, Introduction to Hilbert space, New York, Chelsea, 1951.

2. M. Schreiber, On functions of contractions, to appear.

3. - On absolutely continuous operators, to appear.

4. - Unitary dilations of operators in Hilbert space, Duke Math. J. vol. 23 (1956) pp. 579-594.

5. B. Sz.-Nagy, Sur les contractions de l'espace de Hilbert, Scta Sci. Math. Szeged vol. 15 (1953) pp. 87-92.

6. B. Sz.-Nagy and C. Foias, Sur les contractions de l'espace de Hilbert. III, Acta Sci. Math. Szeged vol. 19 (1958) pp. 26-45.

Cornell University

\section{A SUBSTITUTE FOR LEBESGUE'S BOUNDED CONVERGENCE THEOREM}

\section{NAMIOKA}

1. Lebesgue's bounded convergence theorem has become a powerful tool in the theory of linear topological spaces, and recently, for a treatment of weak convergence of sequences or for a proof of Krein's theorem, the tendency is to use it in an essential way. ${ }^{1}$ The following is a useful substitute for the bounded convergence theorem stated in the language of linear space theory.

THEOREM 1. Let $C$ be a compact (or countably compact) ${ }^{2}$ subset of a (real or complex) linear topological space $E$, and let $\left\{f_{n}\right\}$ be a sequence of continuous linear functionals on $E$ which is uniformly bounded on $C$. If, for each $x$ in $C, \lim _{n} f_{n}(x)=0$, then the same equality holds for every $x$ in the closed convex extension of $C$.

In case $C$ is compact and Hausdorff, the proof of Theorem 1 may run as follows: Let $F$ be the Banach space of all scalar-valued continuous functions on $C$ with the supremum norm; then there is a linear transformation $T$ on the dual $E^{*}$ of $E$ into $F$ defined by the equation $T(f)=f \mid C$. Let $x_{0}$ be a point in the closed convex extension of $C$. Then one can define a bounded functional $\phi$ on the range of $T$

Received by the editors October 20, 1960.

${ }^{1}$ I am indebted to the referee for the remark that, in Dunford and Schwartz [2], Krein's theorem is proved using Riesz-Markoff-Kakutani's theorem but not Lebesgue's bounded convergence theorem. Their proof relies on the theory of integration of vector-valued functions.

${ }^{2}$ A space $X$ is countably compact if each sequence in $X$ has a cluster point. 
by requiring that $\phi(T(f))=f\left(x_{0}\right)$. By the Hahn-Banach extension theorem, $\phi$ can be extended to a bounded functional $\bar{\phi}$ on $F$. Hence there exists a finite Baire measure $\mu$ on $C$ such that $\phi(g)=\int_{c} g d \mu$ for each $g$ in $F$. In particular, for each $f$ in $E^{*}, f\left(x_{0}\right)=\phi(T(f))=\int_{C} T(f) d \mu$ $=\int_{c} f d \mu$. Since the sequence $\left\{f_{n}\right\}$ is uniformly bounded on $C$ and $\lim _{n} f_{n}(x)=0$ for each $x$ in $C$, it follows from the bounded convergence theorem that $\lim _{n} f_{n}\left(x_{0}\right)=\lim _{n} \int_{C f_{n}} d \mu=0$.

The purpose of the present note is to give a proof of Theorem 1 without using either the Riesz-Markoff-Kakutani representation of the dual of the space of continuous functions on a compact Hausdorff space or Lebesgue's bounded convergence theorem. Instead, our proof is based on a theorem (Theorem 3) which is combinatorial in nature. Our approach is related to Banach's beautiful exposition of weak convergence in the annexe of his book (Banach [1]), because the lemma needed to prove Theorem 3 is contained in the proof of Theorem 5 of Banach [op. cit. p. 219]. As an illustration, a sketch of a proof using Theorem 1 of Krein's theorem on weak compactness is included at the end of the note. Our terminology agrees with that of Kelley [5].

2. A nonvoid family $a$ of sets is called an algebra if the union $I$ of $a$ belongs to $a$ and, whenever $A$ and $B$ are members of $a, A \cup B$ and $I \sim A$ (and hence $A \cap B$ and the null set) belong to $a$. A real-valued function $m$ on $a$ is called additive if for any disjoint members $A$ and $B$ of $Q$ it holds that $m(A \cup B)=m(A)+m(B)$. The proof of the following lemma is included here for the sake of completeness.

LeMma 2. Let $m$ be a non-negative real-valued additive function on an algebra of sets $Q$, and let $\left\{A_{k}\right\}$ be a sequence of members of a such that, for some positive number $e, m\left(A_{k}\right)>e$ for all $k$. Then there exists $a$ subsequence $\left\{A_{k_{i}}\right\}$ of $\left\{A_{k}\right\}$ such that $m\left(\cap\left\{A_{k_{i}}: i=1,2, \cdots, n\right\}\right)>0$ for all $n$.

Proof. It is sufficient to prove, under the hypothesis of the lemma, that there exists a subsequence $\left\{A_{k_{j}}\right\}$ of $\left\{A_{k}\right\}$ such that, for some positive number $d, m\left(A_{k_{1}} \cap A_{k_{j}}\right)>d$ for $j=2,3, \cdots$, because then the new sequence $\left\{A_{k_{1}} \cap A_{k_{j}}: j=2,3, \cdots\right\}$ satisfies the hypothesis of the lemma and the lemma will follow by repeating the process.

Let $B_{n}=U\left\{A_{k}: k=1,2, \cdots, n\right\}$; then $\left\{m\left(B_{n}\right)\right\}$ is a bounded nondecreasing sequence of real numbers. Let $a=\lim _{n} m\left(B_{n}\right)$. Then there is a positive integer $r$ such that $a-m\left(B_{r}\right)<e / 2$. Hence, for any integer $k$ greater than $r, m\left(A_{k} \sim B_{r}\right) \leqq m\left(B_{k} \sim B_{r}\right)=m\left(B_{k}\right)-m\left(B_{r}\right)$ $<e / 2$, so that $m\left(A_{k}\right)>e$ implies that $m\left(A_{k} \cap B_{r}\right)>e / 2$, which in turn 
implies that there is an integer $s$ such that $1 \leqq s \leqq r$ and $m\left(A_{s} \cap A_{k}\right)$ $\geqq e / 2 r$. Therefore there is an $s$ such that $m\left(A_{s} \cap A_{k}\right) \geqq e / 2 r$ holds for an infinite number of $k$ 's, and the assertion of the previous paragraph is established. The proof of the lemma is complete.

In the sequel, if $A$ is a finite set, we denote the number of elements in $A$ by $c(A)$.

THEOREM 3. Let $\left\{I_{i}\right\}$ be a sequence of pairwise-disjoint finite nonvoid sets and let $I=\bigcup\left\{I_{i}: i=1,2, \cdots\right\}$. If $\left\{A_{k}\right\}$ is a sequence of subsets of $I$ such that, for some positive number e, $\lim \inf _{i} c\left(A_{k} \cap I_{i}\right) / c\left(I_{i}\right)$ $>e$ for each $k$, then there is a subsequence $\left\{A_{k_{i}}\right\}$ of $\left\{A_{k}\right\}$ such that $\cap\left\{A_{k_{i}}: i=1,2, \cdots, n\right\}$ is infinite for each $n$.

Proof. Let $a$ be the algebra of all subsets of $I$, and for each $i$ define a function $m_{j}$ on $Q$ by $m_{i}(A)=c\left(A \cap I_{i}\right) / c\left(I_{i}\right)$ for an $A$ in $Q$. Then $m_{i}$ is a non-negative real-valued additive function, and $m_{i}(A)$ $\leqq 1$ for all $A$. Hence by Tychonoff's theorem (Kelley [5, p. 143]), the sequence of functions $\left\{m_{i}: i=1,2, \cdots\right\}$ has a cluster point, say $m$, with respect to the topology of pointwise convergence. It is easy to see that $m$ is again a non-negative additive function on $a$ and that, for each $k, m\left(A_{k}\right)>e$. Hence by Lemma 2 , there is a subsequence $\left\{A_{k_{i}}\right\}$ of $\left\{A_{k}\right\}$ such that $m\left(\bigcap\left\{A_{k_{i}}: i=1,2, \cdots, n\right\}\right)>0$ for all $n$. Since $m(A)>0$ implies that $A$ is infinite, the theorem is established.

In the proof above, instead of $a$, we could have used the smallest algebra $a_{0}$ containing the sequence $\left\{A_{i}: i=1,2, \cdots\right\}$. Then by Theorem $C$ of Halmos $\left[4\right.$, p. 23] $a_{0}$ is countable, and the use of Tychonoff's theorem may be replaced by the diagonal process of extracting a converging subsequence (on $a_{0}$ ) of $\left\{m_{i}\right\}$.

In our application of Theorem 3, we use only the fact that, if a sequence $\left\{A_{k}\right\}$ satisfies the condition of Theorem 3 , then there is a subsequence $\left\{A_{k_{i}}\right\}$ such that $\cap\left\{A_{k_{i}}: i=1,2, \cdots, n\right\}$ is nonempty for each $n$. However, since this formally weakened form of Theorem 3 immediately implies Theorem 3 as stated, its apparent weakness is illusory.

3. Proof of Theorem 1. Clearly it is sufficient to prove the theorem under the condition that $C$ is countably compact. Without loss of generality we can assume that $\sup \left\{\left|f_{n}(x)\right|: x \in C\right\} \leqq 1$ for each $n$. Let $\langle C\rangle$ denote the convex extension of the set $C$, and suppose that, for some $x$ in $\langle C\rangle^{-}$, it is false that $\lim _{n} f_{n}(x)=0$. Then, if necessary by taking a subsequence, we can assume that, for some positive number $e,\left|f_{n}(x)\right|>e$ for all $n$. Now, for each $i$, there is an $x_{i}$ in $\langle C\rangle$ such that $\left|f_{n}\left(x_{i}\right)\right|>e$ for each $n \leqq i$, since $x$ belongs to the closure of $\langle C\rangle$. 
It is possible to choose $x_{i}$ in the form: $x_{i}=1 / c\left(I_{i}\right) \sum\left\{x_{a}: a \in I_{i}\right\}$, where $I_{i}$ is a finite index set and $x_{a} \in C$ for each $a$ in $I_{i}$. (It may happen that $x_{a}=x_{b}$ for different $a$ and $b$ in $I_{i}$.) It is also possible to choose index sets so that $I_{i} \cap I_{j}$ is void if $i \neq j$. Let $I=\cup\left\{I_{i}: i=1,2, \cdots\right\}$ and $A_{k}=\left\{a: a \in I\right.$ and $\left.\left|f_{k}\left(x_{a}\right)\right|>e / 2\right\}$. If $i \geqq k$, then

$$
\begin{aligned}
& e<\left|f_{k}\left(x_{i}\right)\right| \leqq 1 / c\left(I_{i}\right) \sum\left\{\left|f_{k}\left(x_{a}\right)\right|: a \in I_{i}\right\} \\
& =1 / c\left(I_{i}\right)\left[\sum\left\{\left|f_{k}\left(x_{a}\right)\right|: a \in A_{k} \cap I_{i}\right\}+\sum\left\{\left|f_{k}\left(x_{a}\right)\right|: a \in I_{i} \sim A_{k}\right\}\right] \\
& \leqq 1 / c\left(I_{i}\right)\left[c\left(A_{k} \cap I_{i}\right)+(e / 2) c\left(I_{i} \sim A_{k}\right)\right] \leqq c\left(A_{k} \cap I_{i}\right) / c\left(I_{i}\right)+e / 2 .
\end{aligned}
$$

Hence, $i \geqq k$ implies that $c\left(A_{k} \cap I_{i}\right) / c\left(I_{i}\right)>e / 2$. In view of Theorem 3 above, there is a subsequence $\left\{A_{k_{i}}\right\}$ of $\left\{A_{k}\right\}$ such that, for each $n$, the intersection $\cap\left\{A_{k_{i}}: i=1,2, \cdots, n\right\}$ contains at least one element say $a_{n}$. Let $y_{n}=x_{a_{n}}$; then $y_{n}$ is in $C$, and, for each $i$ such that $i \leqq n$, it holds that $\left|f_{k_{i}}\left(y_{n}\right)\right|>e / 2$. Let $y_{0}$ be a cluster point in $C$ of the sequence $\left\{y_{n}\right\}$. Then for each $i,\left|f_{k_{i}}\left(y_{0}\right)\right| \geqq e / 2$, but this contradicts $\lim _{n} f_{n}\left(y_{0}\right)=0$ and the proof is complete.

Finally, we give a sketch of a proof of Krein's theorem which asserts that if $A$ is a weakly compact subset of a Banach space $E$, then the closed convex extension $\langle A\rangle^{-}$of $A$ is also weakly compact. By Eberlein's theorem (Eberlein [3]) it suffices to prove that $\langle A\rangle^{-}$is countably compact; hence without loss of generality, $E$ can be assumed to be separable. Let $e$ be the evaluation map of $E$ into $E^{* *}$; then, since $A$ is bounded, the weak* closure $\langle e[A]\rangle^{-}$is weak* compact. Let $\left\{f_{n}\right\}$ be an arbitrary sequence in the unit sphere of $E^{*}$ tending to zero relative to the weak* topology. Regarding $\left\{f_{n}\right\}$ as a sequence of weak* continuous functionals on $E^{* *}$, one concludes from Theorem 1 that $\phi\left(f_{n}\right) \rightarrow 0$ for each $\phi$ in $\langle e[A]\rangle-$. Therefore, since the unit sphere of $E^{*}$ is metrizable (because $E$ is separable), each $\phi$ in $\langle e[A]\rangle^{-}$is weak* continuous on the unit sphere of $E^{*}$ and hence on the whole of $E^{*}$ (for $E$ is complete). It follows that the weak* compact set $\langle e[A]\rangle^{-}$is contained in $e[E]$ and the theorem of Krein follows in turn.

\section{REFERENCES}

1. S. Banach, Théorie des opérations linéaires, Warsaw, 1932.

2. N. Dunford and J. T. Schwartz, Linear operators, Part 1, New York, Interscience Publishers, 1958.

3. W. A. Eberlein, Weak compactness in Banach spaces, Proc. Nat. Acad. Sci. U.S.A. vol. 33 (1947) pp. 51-53.

4. P. R. Halmos, Measure theory, New York, Van Nostrand, 1950.

5. J. L. Kelley, General topology, New York, Van Nostrand, 1955.

Cornell University 